\title{
Fast Orientation Mapping from HARDI
}

\author{
Evren Özarslan ${ }^{1}$, Timothy M. Shepherd ${ }^{2}$, Baba C. Vemuri ${ }^{1}$, \\ Stephen J. Blackband ${ }^{2}$, and Thomas H. Mareci ${ }^{3}$ \\ 1 Department of Computer and Information Science and Engineering, \\ University of Florida, P.O. Box 116120, Gainesville, FL 32611, USA \\ \{evren, vemuri\}@cise.ufl.edu \\ 2 Department of Neuroscience, University of Florida, \\ P.O. Box 100244, Gainesville, FL 32610, USA \\ 3 Department of Biochemistry and Molecular Biology, University of Florida, \\ P.O. Box 116120, Gainesville, FL 32611, USA
}

\begin{abstract}
This paper introduces a new, accurate and fast method for fiber orientation mapping using high angular resolution diffusion imaging (HARDI) data. The approach utilizes the Fourier relationship between the water displacement probabilities and diffusion attenuated magnetic resonance (MR) signal expressed in spherical coordinates. The Laplace series coefficients of the water displacement probabilities are evaluated at a fixed distance away from the origin. The computations take under one minute for most three-dimensional datasets. We present orientation maps computed from excised rat optic chiasm, brain and spinal cord images. The developed method will improve the reliability of tractography schemes and make it possible to correctly identify the neural connections between functionally connected regions of the nervous system.
\end{abstract}

\section{Introduction}

The diffusional attenuation of MR signal has been exploited to characterize diffusional anisotropy in fibrous tissues such as white-matter of the central nervous system (CNS) [1. When the duration of the applied diffusion sensitizing gradients $(\delta)$ is much smaller than the time between the two pulses $(\Delta)$, the fundamental relationship between the MR signal attenuation $E(\mathbf{q})$ and average displacement probabilities $P(\mathbf{R})$ is given by a Fourier integral [2]:

$$
P(\mathbf{R})=\int E(\mathbf{q}) \exp (-2 \pi i \mathbf{q} \cdot \mathbf{R}) d \mathbf{q},
$$

where $\mathbf{R}$ is the displacement vector and $\mathbf{q}$ is the reciprocal space vector defined by $\mathbf{q}=\gamma \delta \mathbf{G} / 2 \pi$, where $\gamma$ is the gyromagnetic ratio and $\mathbf{G}$ is the gradient vector.

In fibrous tissues the orientations specified by large displacement probabilities are expected to coincide with the fiber orientations. One could in principle estimate the values of $P(\mathbf{R})$ by using Eq. 1 and fast Fourier transform (FFT), however, this would require data points all across the space spanned by the diffusion gradients (or $\mathbf{q}$ vectors) necessitating very high gradient strengths and long acquisition times that are difficult to achieve in clinical settings. 
More than a decade ago, Basser et al. introduced diffusion tensor imaging (DTI) 3] that employed a symmetric, positive-definite, second order tensor to characterize the signal attenuation. This model enabled simple measurement and estimation of diffusional anisotropy, and predicted a fiber orientation specified by the principal eigenvector of the diffusion tensor. Despite its modest requirements, DTI has been very successful in most areas of white-matter in the CNS, and enabled the mapping of anatomical connections [4.

DTI assumes a displacement probability characterized by an oriented Gaussian probability distribution function (PDF) whose covariance matrix is proportional to the diffusion tensor. Such a PDF has only one orientational mode and as such can not resolve more than one fiber orientation inside a voxel. This shortcoming of DTI has prompted interest in the development of more sophisticated models. Tuch et al. introduced the HARDI method [5] which suggested that apparent diffusivities could be evaluated along many orientations independently. The result is an angular distribution of diffisuvities, $D(\mathbf{u})$, henceforth refered to as the diffusivity profile. It has been shown that the diffusivity profile has a complicated structure in voxels with orientational heterogeneity, and it is possible to represent the diffusivity profile using a Laplace series (LS) expansion [6, 7]. However, the peaks of the diffusivity profile do not necessarily yield the orientations of the distinct fiber populations. In [8] it was shown that the spherical harmonic transform (SHT) approach could be thought of as a generalization of DTI since the coefficients of the LS (obtained from the SHT of the diffusivity profile) are related to the components of higher order Cartesian tensors. Later Özarslan et al. proposed to use this generalization to generate signal values on the three-dimensional $q$-space, and evaluated a FFT to approximate the displacement probabilities 9]. Jansons and Alexander proposed a method to compute a displacement probability map [10] also from HARDI datasets by enforcing the condition that the probabilities are nonzero only on a spherical shell. Although the results are encouraging, both of the schemes are computationally expensive.

In this work, we introduce a new method, called the diffusion orientation transform (DOT), that describes how the diffusivity profiles can be transformed into probability profiles. Our approach is described in more detail in Ref. [1]. The constructed function is a LS expansion of the displacement PDF at a fixed radius. Our method is robust, fast and does not employ any model fitting. The requirements of our method makes it suitable for the clinical environment.

\section{Theory}

The Fourier transform as given in (Eq. 1) can be expressed in spherical coordinates. This is a consequence of the pointwise convergent expansion of the plane wave in spherical coordinates [12] given by

$$
e^{ \pm 2 \pi i \mathbf{q} \cdot \mathbf{R}}=4 \pi \sum_{l=0}^{\infty} \sum_{m=-l}^{l}( \pm i)^{l} j_{l}(2 \pi q R) Y_{l m}(\mathbf{u})^{*} Y_{l m}(\mathbf{r})
$$


Table 1. $A_{l}(\mathbf{u})$ and $B_{l}(\mathbf{u})$ functions up to $l=6$. In this table, $\beta$ stands for $\beta(\mathbf{u})$

\begin{tabular}{lll}
\hline$l$ & $A_{l}(\mathbf{u})$ & $B_{l}(\mathbf{u})$ \\
\hline 0 & 1 & 0 \\
2 & $-\left(1+6 \beta^{-2}\right)$ & 3 \\
4 & $1+20 \beta^{-2}+210 \beta^{-4}$ & $\frac{15}{2}\left(1-14 \beta^{-2}\right)$ \\
6 & $-\left(1+42 \beta^{-2}+\frac{1575}{2} \beta^{-4}+10395 \beta^{-6}\right)$ & $\frac{105}{8}\left(1-36 \beta^{-2}+396 \beta^{-4}\right)$ \\
\hline
\end{tabular}

where $\mathbf{q}=q \mathbf{u}$ and $\mathbf{R}=r \mathbf{r}$, with $q=|\mathbf{q}|$ and $r=|\mathbf{R}|$. Here $j_{l}(x)$ denotes the $l$-th order spherical Bessel function whereas $Y_{l m}(\mathbf{r})$ are the spherical harmonics. Inserting this expression into Eq. 1, we get

$$
P\left(R_{0} \mathbf{r}\right)=\sum_{l=0}^{\infty} \sum_{m=-l}^{l}(-i)^{l} Y_{l m}(\mathbf{r}) \int d \mathbf{u} Y_{l m}(\mathbf{u})^{*} I_{l}(\mathbf{u}),
$$

where

$$
I_{l}(\mathbf{u})=4 \pi \int_{0}^{\infty} d q q^{2} j_{l}\left(2 \pi q R_{0}\right) \exp \left(-4 \pi^{2} q^{2} t D(\mathbf{u})\right) .
$$

Here $t$ is the diffusion time, $r$ was set to a particular radius $R_{0}$ and it is assumed that signal attenuation along each radial line in $q$-space is dictated by the Stejskal-Tanner relationship [13] where the rate of this attenuation is characterized by the diffusivity profile $D(\mathbf{u})$. Note that the function $P\left(R_{0} \mathbf{r}\right)$ is the probability of finding the particle, initially at the origin, at the point $R_{0} \mathbf{r}$, i.e., we will be interested in the probability values on a sphere of radius $R_{0}$. The integral in Eq. 4 can be evaluated and it is given by

$$
I_{l}(\mathbf{u})=\frac{R_{0}^{l} \Gamma\left(\frac{l+3}{2}\right)}{2^{l+3} \pi^{3 / 2}(D(\mathbf{u}) t)^{(l+3) / 2} \Gamma(l+3 / 2)}{ }_{1} F_{1}\left(\frac{l+3}{2} ; l+\frac{3}{2} ;-\frac{R_{0}^{2}}{4 D(\mathbf{u}) t}\right),
$$

where ${ }_{1} F_{1}$ is the confluent hypergeometric function. Using the recurrence relations of the confluent hypergeometric functions [14] iteratively, these functions can be written in the following form:

$$
I_{l}(\mathbf{u})=A_{l}(\mathbf{u}) \frac{\exp \left(-\beta(\mathbf{u})^{2} / 4\right)}{(4 \pi D(\mathbf{u}) t)^{3 / 2}}+B_{l}(\mathbf{u}) \frac{\operatorname{erf}(\beta(\mathbf{u}) / 2)}{4 \pi R_{0}^{3}},
$$

where

$$
\beta(\mathbf{u})=\frac{R_{0}}{\sqrt{D(\mathbf{u}) t}} .
$$

$A_{l}(\mathbf{u})$ and $B_{l}(\mathbf{u})$ values for functions up to $l=6$ are given in Table 1 . Note that only the even order terms will be included which is a consequence of the antipodal symmetry of the diffusivity profiles as well as the displacement PDFs.

In Figure 1 we plot the square root of $I_{l}$ as a function of $R_{0}$ calculated with double precision using Eq. [6 with $D=1.5 \times 10^{-3} \mathrm{~mm}^{2} / \mathrm{s}$ and $t=25 \mathrm{~ms}$. Very 

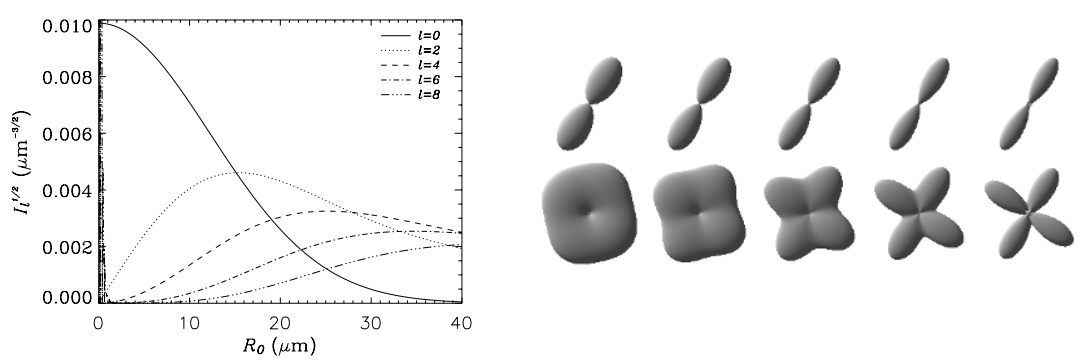

Fig. 1. a. Dependence of the square root of the radial integral, $\sqrt{I_{l}}$, on $R_{0}$. The curve is drawn for $l$ values ranging from 0 to 8 . b. Probability maps estimated on a sphere of radius 8 to $16 \mu \mathrm{m}$ in equal steps of $2 \mu \mathrm{m}$ (from left to right) for voxels with a single orientation (top row) and two distinct orientations (bottom row).

large values taken by the higher order terms near the origin are due to round-off errors. However, this is not a big concern because we will mostly be interested in the values of this function in the $10-20 \mu m$ range. Note that the contribution from higher order terms is rapidly collapsing for $R_{0}$ values in this range.

In order to estimate the probability on the surface of a sphere of radius $R_{0}$, we go back to Eqs. 3 and 4 , and expand $I_{l}(\mathbf{u})$ in Laplace series, i.e.,

$$
I_{l}(\mathbf{u})=\sum_{l^{\prime}=0}^{\infty} \sum_{m^{\prime}=-l^{\prime}}^{l^{\prime}} \alpha_{l l^{\prime} m^{\prime}} Y_{l^{\prime} m^{\prime}}(\mathbf{u}),
$$

where

$$
\alpha_{l l^{\prime} m^{\prime}}=\int Y_{l^{\prime} m^{\prime}}(\mathbf{u})^{*} I_{l}(\mathbf{u}) d \mathbf{u} .
$$

Comparing the integration over $\mathbf{u}$ in Eq. 3 with the expression in Eq. 9, it is easy to see that

$$
P\left(R_{0} \mathbf{r}\right)=\sum_{l=0}^{\infty} \sum_{m=-l}^{l}(-i)^{l} \alpha_{l l m} Y_{l m}(\mathbf{r}),
$$

which is just a LS expansion of $P\left(R_{0} \mathbf{r}\right)$. Note that coefficients of this LS for some $l$ value come from the $l$-th order LS coefficients of $I_{l}(\mathbf{u})$.

\subsection{Implementation Aspects}

In summary, the estimation of the probability of finding the particle at the point $R_{0} \mathbf{r}$ away from the origin involves the following steps:

1. Compute the diffusivity profile $D(\mathbf{u})$ using the Stejskal-Tanner relation.

2. Compute $I_{l}(\mathbf{u})$ using Eq. 5 or 6 with Table 1.

3. Compute $\alpha_{l l m}$, the $l$-th order SHT of $I_{l}(\mathbf{u})$.

4. Evaluate Eq. 10. 
Implementation of the items 1, 2 and 4 above are trivial. Our data acquisition scheme involves sampling the sphere on the vertices of a tessellated icosahedron. This way, 46 or 81 points are sampled on the unit hemisphere from second or third order tessellations respectively. Following [15], we compute the SHT by discretizing the integrals on the sphere with integration weights calculated from the areas of the polygons specified by the dual tessellation. The computation of the $\alpha_{l l m}$ coefficients take 25 to $60 \mathrm{~s}$ for the entire dataset depending on the matrix size and number of sample points using a modest AMD Athlon XP 1800 processor.

\section{Simulations}

We have applied the scheme described above to the simulations of single fiber and crossing fiber systems. The simulations employed the exact form of the MR signal from particles diffusing inside cylindirical boundaries [16. Fig. 10 shows that increasing $R_{0}$ gives rise to the sharpening of the displacement PDFs. This could be predicted from Fig. 11 a which clearly indicates that for small $R_{0}$ the largest contribution comes from $I_{0}$ which upon the SHT forms the isotropic part of the constructed probabilities. Therefore, when $R_{0}$ is small compared to the characteristic length $\sqrt{6 D t}$ associated with the diffusion process and the radii of the cylinders, distribution of probability on the surface becomes more uniform.

We have also computed the probability surfaces for a simulated image of fiber crossing shown in Figure 2. The surfaces are consistent with the underlying known fibrous structure. The circular and linear fiber bundles were chosen so that a distribution of crossing angles is achieved across the region with orientational heterogeneity. We notice that distinct fiber orientations are better resolved when the different fiber bundles make larger angles with each other. Fig. 2a shows the probability profiles when there is no noise added to the signal values. Similar to the simulations in [10], we added Gaussian noise of increasing standard deviation to the real and complex parts of the signal. When the signal intensity in the image with no diffusion weighting is taken to be centered around 1, and Gaussian noise

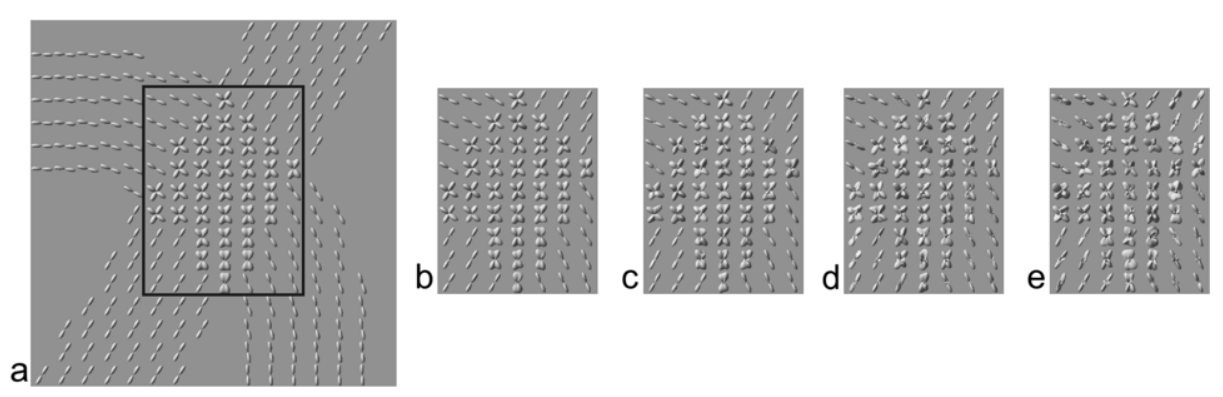

Fig. 2. a. The probability maps from a simulated image of two crossing fiber bundles computed using the DOT method. b-e.Surfaces in the framed area of panel a recomputed under increasing levels of noise. 
of standard deviations 0.02 through 0.08 is added (in equal steps), the probability profiles shown in Fig. 2b-e are obtained. Note that in our standard HARDI protocol, we obtain SNR values in excess of 30 in diffusion weighted scans and about 100 in non-diffusion weighted images. Therefore, in real experiments, one can expect to achieve results that will be of similar or better quality with the image presented in Fig. $2 \mathrm{~b}$.

\section{Results}

In order to test the performance of the DOT method on real tissue, we computed the orientation probabilities on data from three anatomical regions (all from excised rat tissue): optic chiasm, brain and spinal cord. The images were acquired at $17.6 T$ (brain) and $14.1 T$ (spinal cord and optic chiasm) using Bruker Avance imaging systems. Diffusion weighted images were acquired along 81 (brain) and 46 (spinal cord and optic chiasm) directions with a $b$-value of $1500 \mathrm{~mm}^{2} / \mathrm{s}$ (brain and spinal cord) and $1250 \mathrm{~mm}^{2} / \mathrm{s}$ (optic chiasm) along with a single image acquired at $\mathrm{b} \approx 0$. Resolution of the images were $33.6 \times 33.6 \times 200 \mu \mathrm{m}^{3}, 150 \times 150 \times$ $300 \mu \mathrm{m}^{3}, 60 \times 60 \times 300 \mu \mathrm{m}^{3}$ for optic chiasm, brain and spinal cord data respectively. The optic chiasm images were signal averaged to $67.2 \times 67.2 \times 200 \mu \mathrm{m}^{3}$ resolution prior to probability calculations.

For visualization purposes, we have overlaid the orientation surfaces on generalized anisotropy (GA) maps [17] computed from the displacement probabilities. The directionality of the probability profiles on the image plane is readily available from the surfaces. In order to visualize the peakedness through the image plane, we color coded the surface so that as the orientation of the surface varies
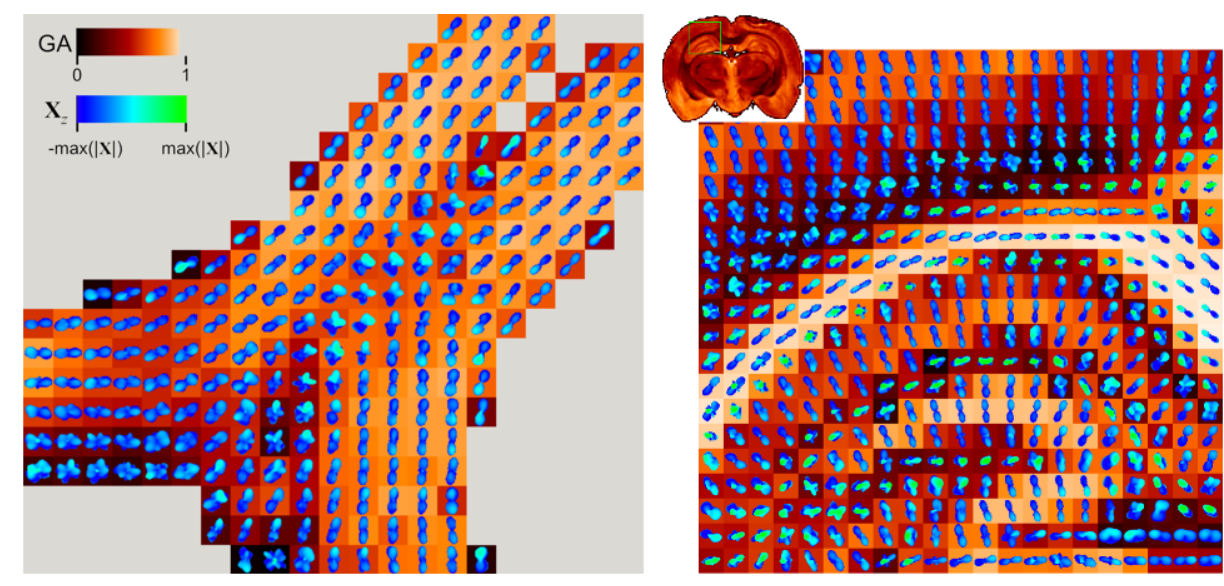

Fig. 3. a. Probability maps computed from a rat optic chiasm dataset. Every other pixel of the image is included for clarity. $\mathbf{b}$. Probability maps computed from a diffusionweighted dataset acquired from an excised rat brain. 


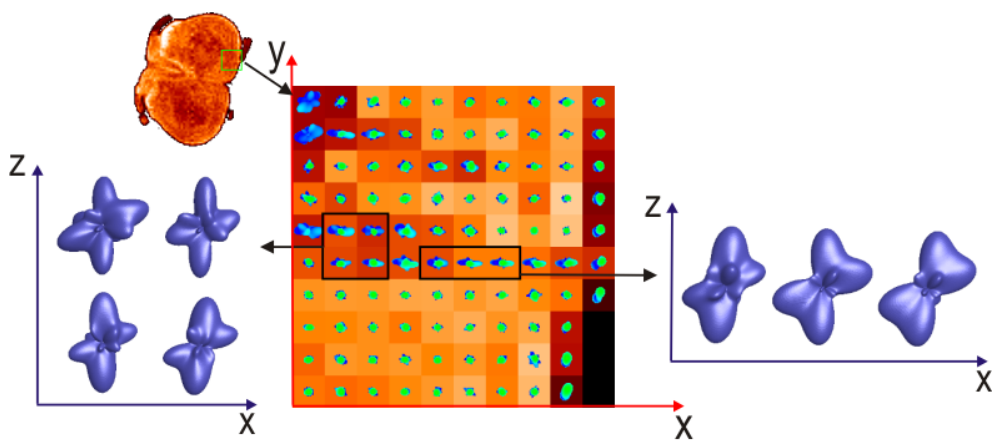

Fig. 4. Probability maps computed from a diffusion-weighted dataset acquired from an excised rat spinal cord

from $-z$-axis to $+z$-axis, its color changes from blue to green. In all calculations $R_{0}$ was set to $16 \mu m$.

Optic chiasm is a distinct white-matter structure with well-known crossing fiber architecture. Therefore, it provides a perfect sample for a validation of the DOT method. Probability maps reconstructed as shown in Figure 3a appear to detect decussating fibers from the temporal visual fields in the center of the optic chiasm. Figure $3 \mathrm{~b}$ shows the displacement probabilities computed from the excised rat brain data set. At the top left is a diffusion-weighted image that shows the selected ROI. The probability maps computed on this ROI demonstrate several interesting cytoarchitectural features of the rat brain. For example, the border between cortex and subcortical white matter contains fibers penetrating from the corpus callosum that cross the radial diffusivities of the cortex.

Finally, we show the probability maps computed from the excised spinal cord data set in Figure 4. Orientation maps from selected pixels of this image are enlarged at the left and rightmost sections of the figure. These surfaces were rotated by $-90^{\circ}$ about the $x$-axis so that the up-and-down direction in the individual surfaces shown in blue correspond to in-and-out direction in the images in red. The magnified surfaces clearly indicate the secondary fiber orientations that represent subtle differences in the connectivity of white matter in those regions.

\section{Conclusion}

The DOT method introduced in this work provides a direct estimation of displacement probability surfaces within each voxel from HARDI data. The technique is robust and fast. It provides a parametric description of the probability surfaces. As a result high resolution probability surfaces can be reconstructed easily from the calculated Laplace series coefficients. The potential applications of our approach include more accurate estimates of anisotropy and fiber orientations that will improve the existing fiber tractography schemes. 
Acknowlegments. This research was supported by the National Institutes of Health Grants R01-NS42075, R01-NS36992 and P41-RR16105, and the National High Magnetic Field Laboratory (NHMFL), Tallahassee.

\section{References}

1. Chenevert, T.L., Brunberg, J.A., Pipe, J.G.: Anisotropic diffusion in human white matter: demonstration with MR techniques in vivo. Radiology 177 (1990) 328-329

2. Callaghan, P.T.: Principles of Nuclear Magnetic Resonance Microscopy. Clarendon Press, Oxford (1991)

3. Basser, P.J., Mattiello, J., LeBihan, D.: MR diffusion tensor spectroscopy and imaging. Biophys J 66 (1994) 259-267

4. Conturo, T.E., Lori, N.F., Cull, T.S., Akbudak, E., Snyder, A.Z., Shimony, J.S., McKinstry, R.C., Burton, H., Raichle, M.E.: Tracking neuronal fiber pathways in the living human brain. Proc Natl Acad Sci 96 (1999) 10422-10427

5. Tuch, D.S., Weisskoff, R.M., Belliveau, J.W., Wedeen, V.J.: High angular resolution diffusion imaging of the human brain. In: Proc. of the 7th Annual Meeting of ISMRM, Philadelphia. (1999) 321

6. Frank, L.R.: Characterization of anisotropy in high angular resolution diffusionweighted MRI. Magn Reson Med 47 (2002) 1083-1099

7. Alexander, D.C., Barker, G.J., Arridge, S.R.: Detection and modeling of nonGaussian apparent diffusion coefficient profiles in human brain data. Magn Reson Med 48 (2002) 331-340

8. Özarslan, E., Mareci, T.H.: Generalized diffusion tensor imaging and analytical relationships between diffusion tensor imaging and high angular resolution diffusion imaging. Magn Reson Med 50 (2003) 955-965

9. Özarslan, E., Vemuri, B.C., Mareci, T.H.: Multiple fiber orientations resolved by generalized diffusion tensor imaging. In: Proc. of the 12th Scientific Meeting of ISMRM. (2004) 89

10. Jansons, K.M., Alexander, D.C.: Persistent angular structure: new insights from diffusion magnetic resonance imaging data. Inverse Problems 19 (2003) 1031-1046

11. Özarslan, E., Shepherd, T.M., Vemuri, B.C., Blackband, S.J., Mareci, T.H.: Resolution of complex tissue microarchitecture using the diffusion orientation transform (DOT). Technical Report TR05-004, Department of CISE, University of Florida, Gainesville, FL (2005)

12. Schwabl, F.: Quantum Mechanics. Springer-Verlag, Berlin (1989)

13. Stejskal, E.O., Tanner, J.E.: Spin diffusion measurements: Spin echoes in the presence of a time-dependent field gradient. J Chem Phys 42 (1965) 288-292

14. Abramowitz, M., Stegun, I.A.: Handbook of Mathematical Functions: With Formulas, Graphs, and Mathmetical Tables. Dover Publications, New York (1977)

15. Ritchie, D.W., Kemp, G.J.L.: Fast computation, rotation, and comparison of low resolution spherical harmonic molecular surfaces. J Comput Chem 20 (1999) 383395

16. Söderman, O., Jönsson, B.: Restricted diffusion in cylindirical geometry. J Magn Reson A (1995) 94-97

17. Özarslan, E., Vemuri, B.C., Mareci, T.H.: Generalized scalar measures for diffusion MRI using trace, variance and entropy. Magn Reson Med 53 (2005) 866-876 